\title{
LRU-PEA: A Smart Replacement Policy for Non-Uniform Cache Architectures on Chip Multiprocessors
}

\author{
Javier Lira $^{1}$, Carlos Molina ${ }^{1,2}$ and Antonio González ${ }^{3}$ \\ ${ }^{1}$ Universitat Politècnica de Catalunya \\ ${ }^{2}$ Universitat Rovira i Virgili \\ ${ }^{3}$ Intel Barcelona Research Center, Intel Labs - UPC \\ javier.lira@ac.upc.edu, carlos.molina@urv.net and antonio.gonzalez@intel.com
}

\begin{abstract}
The increasing speed-gap between processor and memory and the limited memory bandwidth make last-level cache performance crucial for CMP architectures. Non Uniform Cache Architectures (NUCA) have been introduced to deal with this problem. This memory organization divides the whole memory space into smaller pieces or banks allowing nearer banks to have better access latencies than further banks. Moreover, an adaptive replacement policy that efficiently reduces misses in the last-level cache could boost performance, particularly if set associativity is adopted. Unfortunately, traditional replacement policies do not behave properly as they were designed for single-processors. This paper focuses on Bank Replacement. This policy involves three key decisions when there is a miss: where to place a data block within the cache set, which data to evict from the cache set and finally, where to place the evicted data. We propose a novel replacement technique that enables more intelligent replacement decisions to be taken. This technique is based on the observation that some types of data are less commonly accessed depending on which bank they reside in. We call this technique LRU-PEA (Least Recently Used with a Priority Eviction Approach). We show that the proposed technique significantly reduces the requests to the off-chip memory by increasing the hit ratio in the NUCA cache. This translates into an average IPC improvement of $8 \%$ and into an Energy per Instruction (EPI) reduction of 5\%.
\end{abstract}

\section{INTRODUCTION}

The memory system is a pivotal component which can boost or decrease performance dramatically. Chip Multiprocessors (CMPs) typically incorporate large and shared last-level caches (LLCs) with a homogeneous access time. However, the increasing influence of wire delay in cache design means that access latencies to the last-level cache banks are no longer constant [1]. Non-Uniform Cache Architectures (NUCAs) have been proposed [2] to address this problem. A NUCA divides the whole cache memory into smaller banks and allows nearer cache banks to have lower access latencies than more distant banks, thus mitigating the effects of the cache's internal wires. Therefore, each bank behaves as a regular cache and all of them are connected by means of an interconnection network.

When incoming data arrives at a NUCA cache, it is first determined in which bank the new data should be placed by means of a placement policy. Then, the replacement policy must make three decisions considering a set-associative bank: (1) where to insert the new data in the bank, (2) which data to evict from the bank and (3) where to place the evicted data. Notice that a direct-mapped bank only needs to determine what to do with the evicted data. As the number of cores on chip increases, so does the contention caused by applications sharing the LLC. Thus, performance of such systems is heavily influenced by how efficiently the shared cache is managed and an efficient and adaptive replacement policy could boost performance.

One of the key challenges in a replacement policy is choosing the most appropriate data to evict. For this reason, an oracle that knew the future of the program would be the best data eviction policy. Unfortunately, this is not affordable. Thus, most policies already proposed in the literature choose the data to be evicted from the cache on the basis of its history; for example, whether the data has been accessed since its allocation.

In this paper, we propose a novel technique called the LRU-PEA (Least Recently Used with a Priority Evicted Approach) that selects evicted data on the basis of an explicit NUCA data characterization. This approach relies on the traditional LRU scheme whislt also prioritising data within a single bank of the NUCA cache. Furthermore, this mechanism globalizes the replacement decisions that have been taken in a single bank to multiple banks within the NUCA cache.

The remainder of this paper is structured as follows. Section II describes the baseline architecture used in our studies. Section III analyses the characteristics of cache accesses. Section IV presents the experimental methodology. Section V introduces the proposed mechanism, and Section VI analyses its performance and energy consumption. Related work is discussed in Section VII and concluding remarks are given in Section VIII.

\section{BASEline ARCHITECTURE}

As illustrated in Figure 1, the baseline architecture consists of an eight-processor CMP based on that of Beckmann and Wood [3]. The processors are located on the edges of the NUCA cache, which occupies the central part of the chip. Each processor provides the first-level cache memory, composed of two separated caches: one for instructions and one for data. The NUCA cache is the second-level cache memory and is shared by the eight processors. The NUCA cache is divided into 256 banks structured in a $16 \times 16$ mesh that are connected via a $2 \mathrm{D}$ mesh interconnection network. The banks in the NUCA cache are also logically separated into 16 banksets that are either local banks (shaded light in Figure 1) or central banks (shaded dark in Figure 1) 


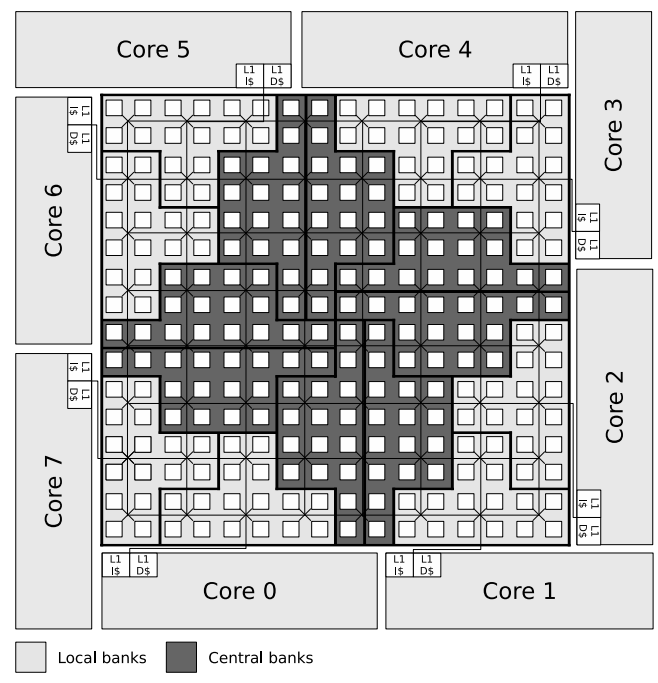

Fig. 1. Baseline architecture layout.

according to their physical distance from the processors. A NUCA model can be characterized by describing how it behaves with the following four policies: Bank Placement Policy, Bank Migration Policy, Bank Access Policy and Bank Replacement Policy. Thus, we can describe the behaviour of the baseline NUCA model for each of the four NUCA policies:

a) Bank Placement Policy: This policy determines in which bank of the NUCA cache memory a data element should be placed when it comes from the off-chip memory or from other caches. We assume that an address can only be mapped to a single bank within the bank-set. Therefore, a data block has 16 possible placements in the NUCA cache (eight local banks and eight central banks). The incoming data from the main memory is placed statically within a bank, whereas the incoming data from the first-level cache is placed in the closest local bank of the requestor core.

b) Bank Migration Policy: This policy is triggered when a hit has been produced in the cache. It determines if a data element is allowed to change its placement from one bank to another bank, which data should be migrated, when this data should be migrated and to which bank it should be moved. We assume gradual promotion. Thus, when there is a hit in a NUCA bank, the accessed data is promoted to a bank that is one-step closer to the processor that has just accessed it.

c) Bank Access Policy: This policy determines the bank-searching algorithm in the NUCA cache memory space. We assume a two-step data search algorithm in the NUCA cache. The first phase of this algorithm broadcasts a request to the local bank that is closest to the processor that launched the search, and to the appropriate eight central banks. If all nine initial requests miss, the request is broadcasted to the remaining seven banks where the address can be placed in the NUCA cache. Only after a request misses all 16 banks will a request be sent off-chip.

d) Bank Replacement Policy: This policy is triggered when a miss has been produced in the cache or when a data block comes from a lower memory level. Once the Bank Placement Policy has determined the bank in which the data should be placed, the Bank Replacement Policy determines how the data is inserted within the bank and which data is evicted from that bank. Therefore, several decisions must be taken, particularly if set-associativity is adopted. For the sake of simplicity, this policy can be split into three more separated sub-policies:

- Data Insertion Policy: This determines the position within the replacement stack of the cache set where the incoming data must be placed. We assume the Most-Recently Used (MRU) mechanism, which is the most commonly used approach in cache organizations.

- Data Eviction Policy: This determines the data that must leave the cache when an incoming data arrives. We assume the Least-Recently Used (LRU) mechanism, which is also the most commonly used approach in all types of cache organizations.

- Data Target Policy: This determines the destination of the data that has been replaced. We assume that the evicted data is sent directly back to the off-chip memory.

\section{Motivation}

Being able to apply a bank migration policy that moves data within the cache is one of the most interesting features of NUCA caches. This enables recently accessed data to be stored close to the requesting core in order to optimize access response times for future accesses. These movements, however, often concentrate the most recently accessed data in the NUCA banks that are next to the cores. As a result, NUCA banks behave differently according to their physical location within the cache. Whereas the banks that are close to the cores (local banks) usually store the most recently accessed data, the banks which are physically located in the centre of the NUCA cache (central banks) store lines that are moving into or leaving local banks, because the bank migration policy implemented uses gradual promotions. In addition to this, bank placement policy also introduces a difference between local banks and central banks. Thus, when data is evicted by a first-level cache, it is relocated to the closest local bank in the NUCA cache. Furthermore, an incoming line from the off-chip memory is statically placed within a NUCA bank.

On the basis of these two features, we classify data in the NUCA cache into four categories: (1) data that has just been promoted (promoted), (2) data that has just been demoted (demoted), (3) data that has just arrived from the off-chip memory (offchip) and (4) data that has just arrived from a first-level cache (L1 replacement).

We analysed the behaviour of the NUCA cache with regard to these four categories, and found that nearly all accesses that were satisfied by local banks were $L 1$ replacement data. However, the vast majority of accesses satisfied by central banks were promoted data.

On the basis of this observation, we propose a novel replacement policy that benefits from the type of data (promoted, demoted, off-chip or L1 replacement) that is most 
commonly accessed from local and central banks. We refer to this mechanism as the LRU-PEA (Least Recently Used with a Priority Eviction Approach).

\section{Experimental Methodology}

\section{A. Simulation Environment}

We use the full-system execution-driven simulator, Simics [4], extended with the GEMS toolset [5]. GEMS provides a detailed memory-system timing model that enables us to model the NUCA cache architecture. Furthermore, it perfectly models the network contention introduced by all simulated mechanisms. The simulated architecture is structured as a single CMP made up of eight UltraSPARC IIIi homogeneous cores. Regarding the memory hierarchy, each core provides a split first-level cache (data and instructions). The second level of the memory hierarchy is the NUCA cache. We used the MOESI token-based coherence protocol to maintain correctness and robustness in the memory system. Table I summarizes the configuration parameters used in our studies. The access latencies of the memory components are based on the models done with the CACTI 6.0 [6] modelling tool, that is the first version of CACTI that provides support for modelling NUCA caches.

\begin{tabular}{|l|l|}
\hline Processors & 8 - UltraSPARC IIIi \\
Frequency & $1.5 \mathrm{GHz}$ \\
\hline Block size & 64 bytes \\
L1 Cache (Instr./Data) & $32 \mathrm{KBytes}, 2$-way \\
L2 Cache (NUCA) & 8 MBytes, 256 Banks \\
NUCA Bank & 32 KBytes, 8-way \\
\hline L1 Latency & 3 cycles \\
NUCA Bank Latency & 4 cycles \\
Router Latency & 1 cycle \\
Avg NUCA Miss Latency & 250 cycles \\
\hline
\end{tabular}

TABLE I

CONFIGURATION PARAMETERS.

We simulated the whole set of applications from the PARSEC benchmark suite [7] with the simlarge input data sets. This suite contains 13 programs from many different areas such as image processing, financial analytics, video encoding, computer vision and animation physics, among others. Regarding the methodology used for the simulations, first we skipped both the initialization and thread creation phases, then we fast-forwarded while warming all caches for 500 million cycles. Finally, we performed a detailed simulation for 200 million cycles.

As performance metric, we used the aggregate number of user instructions committed per cycle, which is proportional to overall system throughput [8].

\section{B. Energy Model}

We deal with a similar energy model to that adopted by Bardine et al [9]. Therefore, we also consider the static and dynamic energy dissipated by the NUCA cache and the additional energy required to access the off-chip memory. The total energy dissipated by the NUCA cache is the sum of all three components:

$$
E_{\text {total }}=E_{\text {static }}+E_{\text {dynamic }}+E_{\text {off-chip }}
$$

To obtain the static energy $\left(\mathrm{E}_{\text {static }}\right)$, the NUCA cache is modelled by means of the CACTI 6.0 tool [6]. The dynamic energy $\left(\mathrm{E}_{\text {dynamic }}\right)$ is modelled by the GEMS toolset [5] which uses the Orion simulator to determine the energy per bank access, the energy required to transmit a flit on the network link and the energy required to switch a flit through a network switch. The extra network traffic introduced by our proposal is also considered and perfectly modeled by the simulator.

The energy dissipated by the off-chip memory is determined using the Micron System Power Calculator [10] with a modern DDR3 system (4GB, 8DQs, Vdd:1.5v, 333 $\mathrm{MHz}$ ). Our evaluation of the off-chip memory focuses on the energy dissipated during active cycles; this energy is isolated it from the background energy. From our results, we conclude that the average energy of each access is $550 \mathrm{pJ}$.

We analysed the consumption results using Energy per instruction (EPI) [11] as our metric. This metric is independent of the amount of time required to process an instruction and is ideal for throughput performance.

\section{LRU WITH PRIORITY EVICTION APPROACH (LRU-PEA)}

As described in Section II, a Bank Replacement Policy can be divided into the following three sub-policies: data insertion policy, data eviction policy and data target policy. In this section we introduce the Least Recently Used with Priority Eviction Approach (LRU-PEA) replacement policy. This policy focuses on optimizing the performance of applications on a CMP-NUCA architecture by analyzing data behaviour within the NUCA cache and trying to keep the most accessed data in cache as long as possible. In order to describe how this policy works, we describe separately the two sub-policies that the LRU-PEA modifies: data eviction policy and data target policy. With regard to data insertion policy, we assume the MRU mechanism (same as baseline).

\section{A. Data Eviction Policy}

The LRU-PEA statically prioritises the previously defined categories (promoted, demoted, offchip and $L 1$ replacement). However, the two groups of banks are too different, so the LRU-PEA defines the prioritisation for both local and central banks. Having a static prioritisation, however, could cause the highest-category data to monopolize the NUCA cache, or even cause a simple data block to stay in the cache forever. In order to avoid these situations, we restrict the category comparison to the two last positions in the LRU-stack. In this way, even data with the lowest category will stay in the cache until it arrives at the LRU-1 position in the LRU-stack.

Figure 2 gives an example of how the LRU-PEA scheme works. First, we define the prioritisation of the data categories. For instance, the prioritisation of the example is as follows (see Figure 2(a)): 1) L1 Replacement, 2) Promoted, 3) Offchip and 4) Demoted. When the LRU-PEA eviction policy is applied, the last two positions of the LRU-stack compete to find out which one is going to be evicted (see Figure 2(b)). Thus, we can compare their categories. If they are different, the data with the lower 


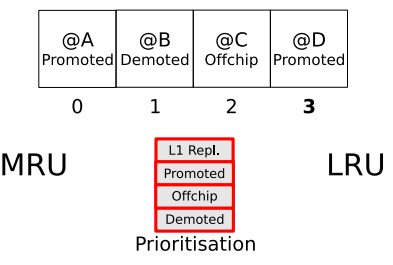

(a)

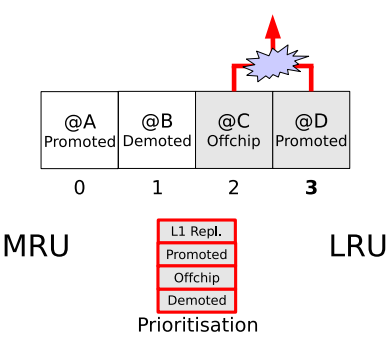

(b)

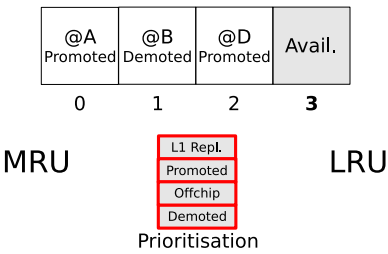

(c)

Fig. 2. LRU-PEA scheme. (a) Initial state of the LRU-stack. (b) The last two positions of the LRU-stack compete to avoid being evicted. (c) The lowest category data has been evicted.

category is evicted. But, if both have the same category, the line that currently occupies the LRU position is evicted. Finally, the data that has not been evicted updates its position within the LRU-stack (see Figure 2(c)).

By analysing NUCA cache behaviour, we found that local banks access L1 Replacement data more frequently, whereas central banks mainly access promoted data. Thus, we evaluate the LRU-PEA using the prioritisation described in Table II.

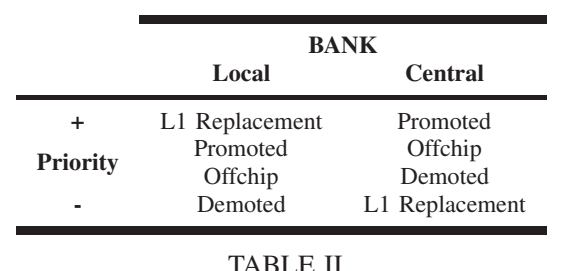

PRIORITISATION FOR LRU-PEA.

We have also experimentally observed that modifying the priority order of the lower categories does not introduce significant differences in terms of performance.

\section{B. Data Target Policy}

There are two key issues when a Dynamic-NUCA (D-NUCA) architecture [2] is considered: 1) a single data can be mapped in multiple banks within the NUCA cache, and 2) the migration process moves the most accessed data to the banks that are closer to the requesting cores. Therefore, bank usage in a NUCA cache is heavily imbalanced, and a capacity miss in a heavy-used NUCA bank could cause constantly accessed data to be evicted from the NUCA cache, while other NUCA banks are storing less frequently accessed data. The LRU-PEA addresses this problem by defining a data target policy that allows the replacement decision that has been taken in a single bank to be spread to all banks in the NUCA cache where evicted data can be mapped.

We propose Algorithm 1 as a data target policy for the LRU-PEA. The main idea of this algorithm is to find a NUCA bank whose victim data belongs to a lower priority category than that which is currently

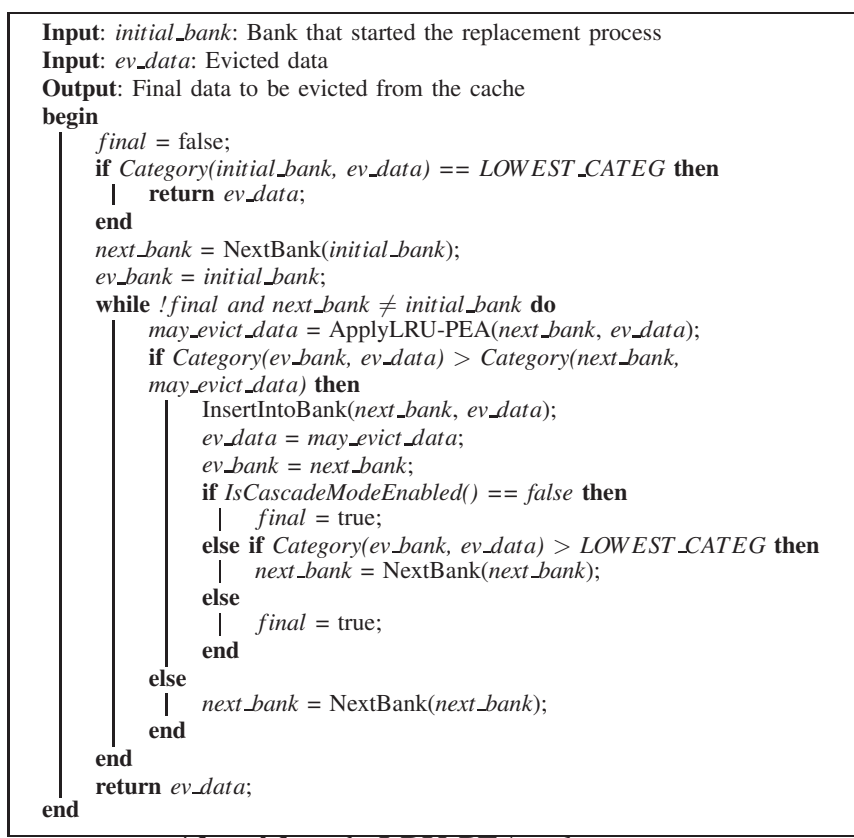

Algorithm 1: LRU-PEA scheme

being evicted. In this way, while the target NUCA bank is not found, all NUCA banks where the evicted data can be mapped are sequentially accessed in an statically defined order. In our evaluation we use the following order: Local_Bank_Core ${ }_{i} \rightarrow$ Central_Bank_Core $_{i}$ $\rightarrow$ Local_Bank_Core $_{i+1} \rightarrow$ Central_Bank_Core $_{i+1} \rightarrow \ldots$

The algorithm finishes when one of the following occurs: 1) the evicted data belongs to the lowest priority category, 2) all NUCA banks where the evicted data can be mapped have been already visited, and 3) the evicted data has been relocated to another NUCA bank. Then, whether the evicted data could not be relocated to other bank into the NUCA cache, it is written back to the upper-level memory.

By using sequential access, however, the accuracy of the LRU-PEA is restricted to the NUCA banks that have been visited before finding a target bank. To address this problem, we introduce the on cascade mode. When this mode is enabled, the algorithm does not finish when the evicted data 


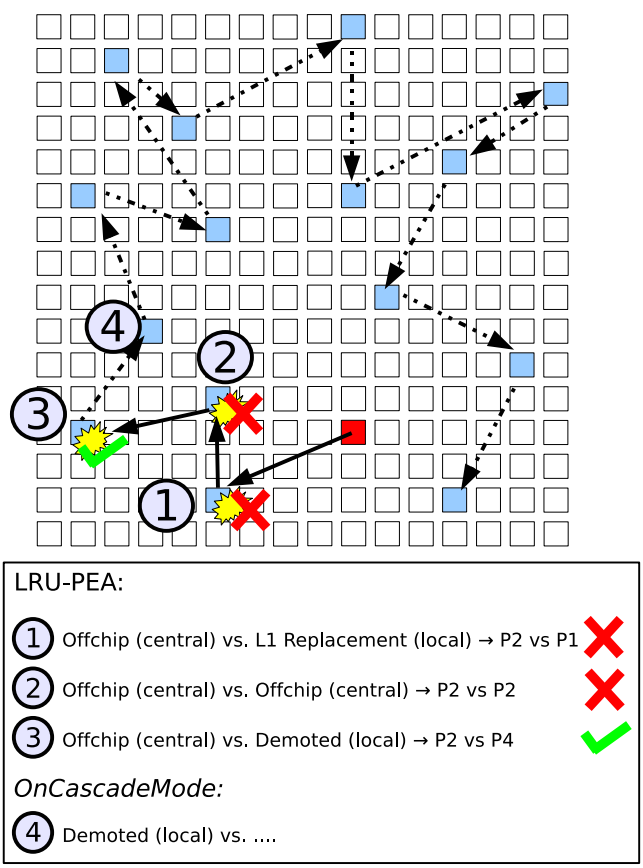

Fig. 3. Example of how LRU-PEA behaves.

finds a target bank. Instead, it uses the data that has been evicted from the target bank as evicted data. Thus, after visiting all NUCA banks we can assure that the current evicted data belongs to the current lowest priority category. In Section VI, we consider both configurations, with the on cascade mode enabled and disabled.

Figure 3 shows an example of how the LRU-PEA's data target policy works. In this example, the algorithm starts in a central bank and the evicted data belongs to the Offchip category, so the priority of the evicted data is 2 (see Table II). First, the algorithm checks whether the evicted data can be relocated in the local bank of the next core (step 1 in Figure 3 ). However, the priority of the victim data in the current bank is higher than the evicted data, so the LRU-PEA tries to relocate the evicted data into the next bank. In the second step, it visits another central bank. In this case, the category of the victim data in the current bank is the same as the evicted data, and so next bank needs to be checked. Finally, in the third step, the category of the evicted data has higher priority than the one of the victim data of the current bank. Thus, the evicted data is relocated to the current bank. If the on cascade mode is enabled, the algorithm continues with the 4th step (see Figure 3), but uses the data that has been evicted from the current bank as evicted data. Otherwise, this data is directly evicted from the NUCA cache and sent back to the upper-level memory.

\section{Additional Hardware}

This mechanism requires the introduction of some additional hardware to the NUCA cache. In order to determine the data's category, we add two bits per line (there are four categories). Then, assuming that 8 MByte NUCA cache described in Section IV is used, LRU-PEA will need to add 32 KBytes, which is less than $0.4 \%$ of the

\begin{tabular}{lccc} 
& \multirow{2}{*}{ No Cascade } & \multicolumn{2}{c}{ Cascade Enabled } \\
\cline { 3 - 4 } & & Direct & Provoked \\
\hline 1 message & 64 & 54 & 20 \\
2 messages & 12 & 7 & 7 \\
3 messages & 4 & 2 & 4 \\
4 messages & 3 & 2 & 4 \\
5 messages & 3 & 2 & 3 \\
6 messages & 2 & 1 & 4 \\
7 messages & 2 & 1 & 3 \\
8 messages & 2 & 1 & 4 \\
9 messages & 1 & 1 & 3 \\
10 messages & 1 & 1 & 4 \\
11 messages & 1 & 1 & 3 \\
12 messages & 1 & 1 & 6 \\
13 messages & 1 & 1 & 6 \\
14 messages & 1 & 1 & 30 \\
15 messages & 3 & 21 & - \\
\hline \multicolumn{4}{c}{ Values in percentage $(\%)$} \\
\hline
\end{tabular}

TABLE III

NUMBER OF EXTRA MESS AGES INTRODUCED BY BOTH CONFIGURATIONS OF LRU-PEA TO SATISFY REPLACEMENTS.

hardware overhead. Furthermore, the proposed mechanism can be easily implemented without significant complexity.

\section{RESUlTS AND ANALYSIS}

This section analyses the impact of assuming the LRU-PEA as bank replacement policy. The LRU-PEA takes advantage of on-chip network introduced by CMPs to provide a sophisticated algorithm that allows the globalization of the replacement decisions that have been taken in a single bank. Although this approach may increase contention in the on-chip network, it is perfectly modelled in our simulator. Table III shows the average number of extra messages introduced by the LRU-PEA to satisfy a single replacement. When the on cascade mode is disabled, the communication overhead introduced by LRU-PEA is very low. On average, close to $80 \%$ of replacements are satisfied by introducing up to 3 extra messages into the on-chip network. By enabling the on cascade mode, however, a significant percentage of replacements introduce the maximum number of messages into the network (the number of banks where the evicted data can be mapped minus one). This difference between the two modes can be explained by the high-accuracy provided by the LRU-PEA when the on cascade mode is enabled. In general, data in NUCA banks has higher priority, and it is much more difficult to find a victim data with lower priority than the evicted data. In the following sections we analyse how the LRU-PEA behaves in terms of performance and energy consumption.

\section{A. Performance Analysis}

Figure 4 shows the IPC improvement achieved when using the LRU-PEA as bank replacement policy in the NUCA cache. On average, we find that the LRU-PEA increases IPC by $8 \%$ with respect to the baseline architecture if the on cascade mode is enabled, and by $7 \%$ when it is disabled. In general, we find that the LRU-PEA significantly 


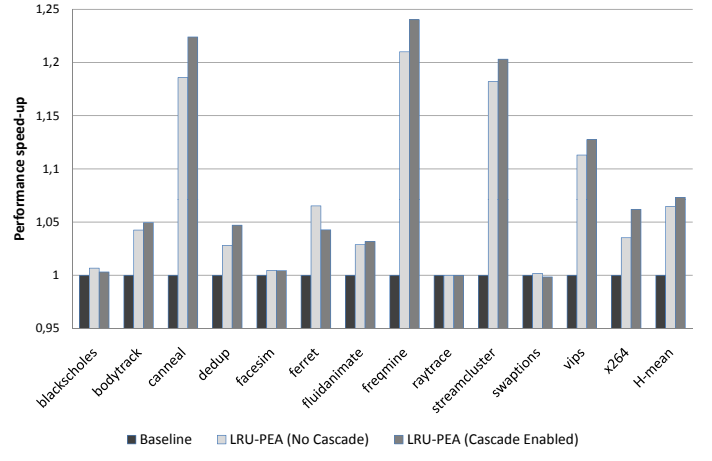

Fig. 4. IPC improvement with LRU-PEA.

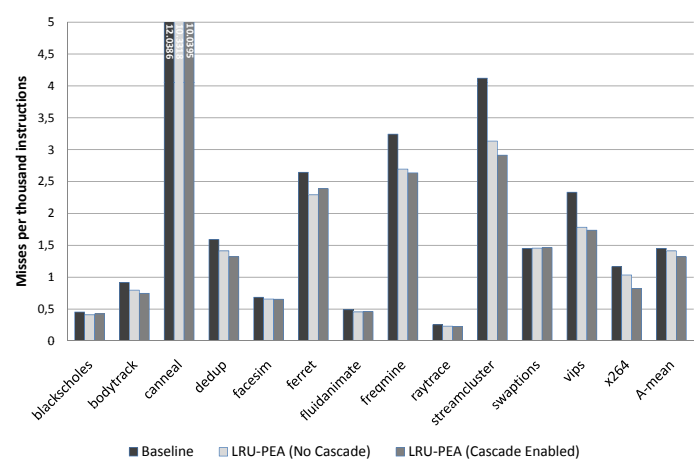

Fig. 5. Misses per thousand instructions with LRU-PEA.

improves IPC with most PARSEC applications, obtaining a near 20\% improvement in three of them (canneal, freqmine and streamcluster). On the other hand, 4 of the 13 PARSEC applications do not show perfomance benefits when using the LRU-PEA (blackscholes, facesim, raytrace and swaptions). We also observe that although the LRU-PEA does not significantly improve performance in some of the PARSEC applications, it is not harmful to performance either.

Figure 5 shows the NUCA misses per 1000 instructions (MPKI) with the three evaluated configurations: baseline, LRU-PEA and LRU-PEA with on cascade mode enabled. On average, we observed a significant reduction in MPKI when using the LRU-PEA, and even more when the on cascade mode is enabled. In general, we found that PARSEC applications that provide performance improvements, also significantly reduce MPKI. Moreover, we saw that canneal, freqmine and streamcluster (the applications that provide the highest IPC improvement with LRU-PEA) also have the highest MPKI. In contrast, applications with an MPKI close to zero do not usually improve performance when the LRU-PEA is used.

Regarding those applications where the LRU-PEA does not improve performance, blackscholes and swaptions are financial applications with small working sets, so their cache requirements are restricted. On the other hand, raytrace and facesim have very big working sets, but they are computationally intensive and mainly exploit temporal locality.

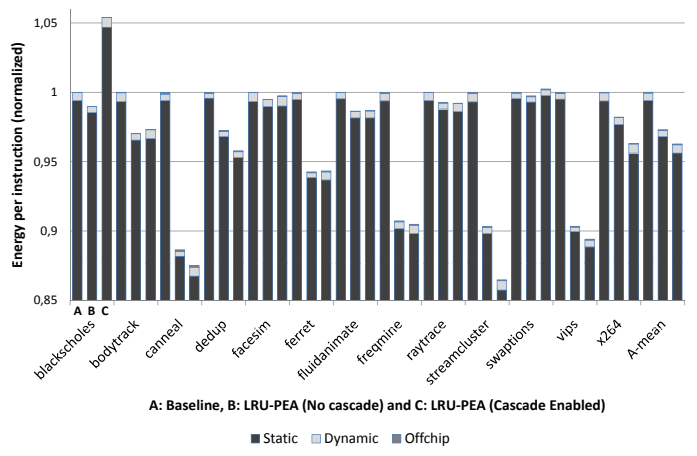

Fig. 6. Normalized average energy consumed per each executed instruction.

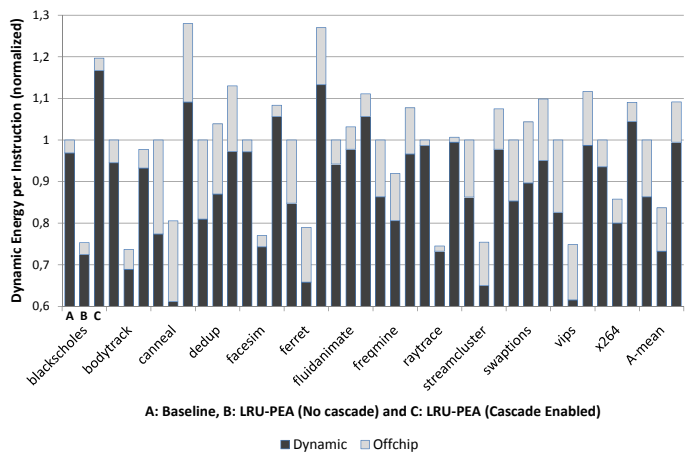

Fig. 7. Normalized average dynamic energy consumed per each instruction.

\section{B. Energy Consumption Analysis}

The energy consumption is analysed by using the Energy per Instruction (EPI) metric. Figure 6 shows that, on average, the LRU-PEA reduces the energy consumed per each instruction compared to the baseline architecture by 5\% for both configurations (with and without the on cascade mode enabled). In particular, the LRU-PEA significantly reduce energy consumption in PARSEC applications with large working sets, such as canneal, freqmine and streamcluster. Moreover, we observed that, with the exception of blackscholes and swaptions, EPI was always reduced by the LRU-PEA.

As we can see in Figure 6, EPI is heavily influenced by static energy. Figure 7 shows the normalized EPI without taking into consideration the static energy consumed. We find that when on cascade mode is enabled, the dynamic energy consumed is $10 \%$ higher than in the baseline configuration. However, the LRU-PEA with on cascade mode disabled still reduces EPI by more than $15 \%$. This difference between the two LRU-PEA modes corresponds to the number of extra messages introduced into the on-chip network by each of them (see Table III).

Finally, we highlight that although LRU-PEA increases the on-chip network contention, the average energy consumed per instruction is still reduced due to the significant performance improvement that this mechanism provides.

\section{RELATED WORK}

Replacement policy brings together two decisions that can be seen as two more policies: data insertion and data eviction. 
The former decides where to place data and the latter decides which data is replaced. Traditionally, caches use the Most Recently Used (MRU) algorithm to insert data and the Least Recently Used (LRU) algorithm to evict data [12], [13].

Modifications to the traditional LRU scheme have been also proposed. Wong and Bauer [14] modified the standard LRU to maintain data that exhibited higher temporal locality. Alghazo et al. [15] proposed a mechanism called SF-LRU (Second-Chance Frequency LRU). This scheme combines both the recentness (LRU) and frequency (LFU) of blocks to decide which blocks to replace. Dybdahl et al. [16] also proposed another LRU approach based on frequency of access in shared multiprocessor caches. Kharbutli and Solihin [17] proposed a counter-based L2 cache replacement. This approach includes an event counter with each line that is incremented under certain circumstances. The line can then be evicted when this counter achieves a certain threshold.

Recently, several papers have revisited data insertion policy. Qureshi et al. [18] propose Line Distillation, a mechanism that tries to keep frequently accessed data in a cache line and to evict unused data. This technique is based on the observation that, generally, data is unlikely to be used in the lowest priority part of the LRU stack. They also proposed LIP (LRU Insertion Policy), which places data in the LRU position instead of the MRU position [19].

Kim et al. [2] introduced the concept of Non-Uniform Cache Architecture (NUCA). They observed that the increase in wire delays would mean that cache access times were no longer constant. Instead, latency would become a linear-function of the line's physical location within the cache. From this observation, several NUCA architectures were designed by partitioning the cache into multiple banks and using a switched network to connect these banks. However, the introduction of CMP architectures posed additional challenges to the NUCA architecture and this led Beckmann and Wood [3] to analyse NUCA for CMP. Recent studies have explored policies for bank placement [20], bank migration [21], bank access [22] and bank replacement [2] in NUCA caches. None of these studies properly addresses bank replacement policy in a CMP environment.

\section{CONCLUSIONS}

The increasing gap between processor and memory speed and the limited memory bandwidth make last-level cache performance crucial for CMP architectures. Reducing last-level cache misses, therefore, will provide significant performance benefits. In this paper we propose a novel alternative to the traditional LRU replacement policy. It aims to make more intelligent replacement decisions by protecting the cache lines that are likely to be reaccessed. On average, LRU-PEA replacement policy improves IPC by $8 \%$ compared to the baseline configuration, and reduces energy consumption per instruction by $5 \%$.

In conclusion, in this paper we demonstrate that minimal modifications to the bank replacement policy to enable more intelligent eviction and target decisions will result in significant performance and consumption benefits.

\section{ACKNOWLEDGEMENTS}

This work is supported by the Spanish Ministry of Science and Innovation (MCI) and FEDER funds of the EU under the contracts TIN 2007-61763 and TIN 2007-68050-C03-03, the Generalitat de Catalunya under grant 2005SGR00950, and Intel Corporation. Javier Lira is supported by the MCI under FPI grant BES-2008-003177.

\section{REFERENCES}

[1] V. Agarwal, M. S. Hrishikesh, S. W. Keckler, and D. Burger, "Clock rate vs. ipc: The end of the road for conventional microprocessors," in Proceedings of the 27th International Symposium on Computer Architecture, 2000.

[2] C. Kim, D. Burger, and S. W. Keckler, "An adaptive, non-uniform cache structure for wire-delay dominated on-chip caches," in Proceedings of the 10th International Conference on Architectural Support for Programming Languages and Operating Systems, 2002.

[3] B. M. Beckmann and D. A. Wood, "Managing wire delay in large chip-multiprocessor caches," in Proceedings of the 37th International Symposium on Microarchitecture, 2004.

[4] P. S. Magnusson, M. Christensson, J. Eskilson, D. Forsgren, G. Hallberg, J. Högberg, F. Larsson, A. Moestedt, and B. Werner, Simics: A Full System Simulator Platform. Computer, 2002, vol. 35-2, pp. 50-58.

[5] M. M. K. Martin, D. J. Sorin, B. M. Beckmann, M. R. Marty, M. Xu, A. R. Alameldeen, K. E. Moore, M. D. Hill, and D. A. Wood, "Multifacet's general execution-driven multiprocessor simulator (gems) toolset," in Computer Architecture News, Sept. 2005.

[6] N. Muralimanohar, R. Balasubramonian, and N. P. Jouppi, "Optimizing nuca organizations and wiring alternatives for large caches with cacti 6.0," in Proceedings of the 40th Annual IEEE/ACM International Symposium on Microarchitecture, 2007.

[7] C. Bienia, S. Kumar, J. P. Singh, and K. Li, "The parsec benchmark suite: Characterization and architectural implications," in Proceedings of the International Conference on Parallel Architectures and Compilation Techniques, 2008.

[8] T. F. Wenisch, R. E. Wunderlich, M. Ferdman, A. Ailamaki, B. Falsafi, and J. C. Hoe, "Simflex: Statistical sampling of computer system simulation," IEEE Micro, vol. 26, no. 4, pp. 18-31, 2006.

[9] A. Bardine, P. Foglia, G. Gabrielli, and C. A. Prete, "Analysis of static and dynamic energy consumption in nuca caches: Initial results," in Proceedings of the 2007 Workshop on Memory Performance: Dealing with Applications, Systems and Architecture, 2007.

[10] Micron system power calculator. [Online]. Available: http

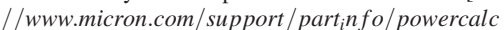

[11] E. Grochowski, R. Ronen, J. Shen, and H. Wang, "Best of both latency and throughput," in Proceedings of the 22nd International Conference on Computer Design, 2004.

[12] L. A. Belady, "A study of replacement algorithms for virtual-storage computer," IMB Systems Journal, vol. 5, no. 2, 1966.

[13] A. J. Smith, "Cache memories," ACM Computing Surveys, vol. 14, no. 3, 1982.

[14] W. Wong and J. Baer, "Modified lru policies for improving second-level cache behavior," in Proceedings of the 6th International Symposium on High-Performance Computer Architecture, 2000.

[15] J. Alghazo, A. Akaaboune, and N. Botros, "Sf-lru cache replacement algorithm," in Records of the International Workshop on Memory Technology, Design and Testing, 2004.

[16] H. Dybdahl, P. Stenström, and L. Natvig, "An lru-based replacement algorithm augmented with frequency of access in shared chip-multiprocessor caches," Computer Architecture News, vol. 35, 2007.

[17] M. Kharbutli and Y. Solihin, "Counter-based cache replacement algorithms," in Proceedings of the 23rd International Conference on Computer Design, 2005.

[18] M. K. Qureshi, M. A. Suleman, and Y. N. Patt, "Line distillation: Increasing cache capacity by filtering unused words in cache lines," in Proceedings of the 13th International Symposium of High-Performance Computer Architecture, 2007.

[19] M. K. Qureshi, A. Jaleel, and Y. N. Patt, "Adaptive insertion policies for high-performance caching," in Proceedings of the 34th International Symposium on Computer Architecture, 2007.

[20] J. Huh, C. Kim, H. Shafi, L. Zhang, D. Burger, and S. W. Keckler, "A nuca substrate for flexible cmp cache sharing," in Proceedings of the 19th ACM International Conference on Supercomputing, 2005.

[21] M. Kandemir, F. Li, M. J. Irwin, and S. W. Son, "A novel migration-based nuca design for chip multiprocessors," in Proceedings of the ACM/IEEE conference on Supercomputing, 2008.

[22] N. Muralimanohar and R. Balasubramonian, "Interconnect design considerations for large nuca caches," in Proceedings of the 34th International Symposium on Computer Architecture, 2007. 